\title{
Philosophiques
}

\section{B. Cassin, (Éditrice), Positions de la sophistique, Paris, Vrin, 1986, 338 pages.}

\section{Louis-André Dorion}

Volume 19, numéro 1, printemps 1992

URI : https://id.erudit.org/iderudit/027178ar

DOI : https://doi.org/10.7202/027178ar

Aller au sommaire du numéro

Éditeur(s)

Société de philosophie du Québec

ISSN

0316-2923 (imprimé)

1492-1391 (numérique)

Découvrir la revue

Citer ce compte rendu

Dorion, L.-A. (1992). Compte rendu de [B. Cassin, (Éditrice), Positions de la sophistique, Paris, Vrin, 1986, 338 pages.] Philosophiques, 19(1), 134-139.

https://doi.org/10.7202/027178ar d'utilisation que vous pouvez consulter en ligne.

https://apropos.erudit.org/fr/usagers/politique-dutilisation/ 
B. CASSIN, (Éditrice), Positions de la sophistique, Paris, Vrin, 1986, 338 pages.

\author{
par Louis-André Dorion
}

Ce volume rassemble plusieurs communications présentées à l'occasion de colloque sur la sophistique qui s'est tenu à Cerisy en 1984. Nous avons déjà traité (cf. Dialogue, vol. 26, n¹, 1987, pp. 196199) du recueil Le plaisir de parler, lequel réunissait celles des communications de ce colloque qui s'intéressaient davantage à la postérité et aux avatars du discours sophistique qu'à la sophistique proprement dite. La très grande majorité des dix-huit études qui constituent le présent recueil se penchent principalement sur la "première sophistique", soit celle qui connut son apogée dans le dernier quart du Ve siècle avant J.C.

G. B. Kerferd, le maître actuel des études sur la sophistique, insiste sur le fait que Platon ne désavoue pas totalement les procédés mis au point par les sophistes. Ainsi en est-il, notamment, de l'antilogike tekhne, dont la principale constituante est le fameux elenchos ("réfutation"). Platon reconnaît sans peine que l'elenchos, dont la principale vertu est de mettre à jour une contradiction dans l'argumentation de l'adversaire, est un outil indispensable pour qui veut "déblayer le terrain"; mais il reproche aux sophistes de s'en tenir uniquement au moment négatif de l'elenchos et de ne pas mettre en œuvre les ressources dialectiques qui permettent d'atteindre la vérité ( Le sophiste vu par Platon: un philosophe imparfait » 13-25). 
Auteur de traductions remarquées du Gorgias et de l'Euthydème, M. Canto signe ici une étude d'une rare densité sur les réfutations sophistique et philosophique ("Politiques de la réfutation. Entre chien et loup: le philosophe et le sophiste ", 27-51). À l'instar de Kerferd, Canto rappelle que la réfutation est commune au sophiste et au philosophe; mais alors que, pour Kerferd, le dialecticien qui maîtrise la pratique de l'elenchos peut très bien se passer du sophiste, Canto croit au contraire que les pratiques sophistiques et philosophiques sont intimement liées dans la mesure où le discours philosophique ne peut avoir un effet réel qu'en se démarquant du sophiste, mais que, pour ce faire, il doit précisément utiliser la méthode de l'elenchos, qui est la marque de commerce du sophiste. D'où le constat de ce paradoxe: «En dehors de la réfutation, commune au sophiste et au philosophe, il n'y a donc pas moyen de séparer l'un de l'autre " (34). On reprochera à Canto de n'être pas très sensible aux différentes valeurs du terme elenchos; c'est en effet une erreur de croire que ce terme a toujours le sens de "réfutation ". Ainsi l'expression elenchos tou biou (45) n'aurait pas le sens de « réfutation de la vie "; cf. aussi Lois (X, 890e6-891a2); Lysias (II, 47), Démosthène (IV, 15). De plus, le traitement du rapport entre l'elenchos et le sentiment de honte (48-49) comporte une importante lacune en ce qu'il omet de signaler que le sens homérique d'elenchos est précisément " honte " (Iliade, XI, 313). Faut-il, dans ces conditions, considérer le rapport explicite que Platon établit entre l'elenchos et la honte (cf. Sophiste $230 \mathrm{~b}-\mathrm{d}$ ) comme le vestige d'une conception ancienne, ou bien ne faut-il y voir rien de plus qu'une pure coïncidence? La question demeure ouverte.

Selon A. Soulez, la dimension pragmatique du discours sophistique n'avait pas échappé à Platon; et, paradoxalement, c'est afin de la mieux combattre que Platon, dans ses dialogues, accorde la parole à la pragmatique sophistique ("Le dire comme acte du sophiste ou: intervention et répudiation par Platon de la pragmatique sophistique ", 53-73).

Auteur d'un intéressant commentaire de l'Euthydème (Le philosophe et son double, Paris, Vrin, 1984), M. Narcy s'emploie à montrer, à partir de trois textes (Théétète, Sophiste et Métaphysique $\Gamma$ ), comment Platon et Aristote se sont efforcés de rendre inopérant le discours sophistique, dont la figure emblématique est Protagoras ("A qui la parole? Platon et Aristote face à Protagoras ", 75-90). L'analyse la plus convaincante est sans contredit celle de Mét. $\Gamma$ : la "stratégie " mise en œuvre par Aristote contre le discours sophistique repose tout entière sur la "contrainte" de la signification, ou, en d'autres mots, sur l'équivalence établie entre "dire quelque chose " et "signifier quelque chose".

Pour R. Sprague, les apories qui avaient pour conséquence de nier l'existence de certains processus, tels l'apprentissage, le mouvement et le changement, ont agi sur Platon et Aristote comme de 
véritables stimuli, en ce sens qu'elles les ont incités à mettre au point des doctrines qui permettent de rendre compte plus finement de ces mêmes processus ( A Apories sophistiques et description du processus ", 91-104). Ainsi la doctrine de l'acte et de la puissance est-elle la réponse d'Aristote à plusieurs de ces apories dont l'inspiration est vraisemblablement éléatique, mais dont la mise en forme, elle, est sans doute le fait des Mégariques.

Dans le dernier chapitre des Réfutations Sophistiques, Aristote raille l'enseignement de ses prédécesseurs, dont Gorgias, et il s'attribue, non sans emphase, tout le mérite d'avoir jeté le premier les bases d'une authentique tekhne dialectique. Mais en quoi consistent au juste les logoi enseignés par Gorgias? Au terme de fort instructifs recoupements avec des textes de Platon, Isocrate et Alcidamas, C. Nathali est en mesure d'affirmer que ces logoi n'étaient pas des discours destinés à être appris par cœur, mais bien des loci communes, c'est-à-dire des arguments qu'il était possible d'utiliser en plusieurs occasions ("Aristote et les méthodes d'enseignement de Gorgias. Réfutations sophistiques 34, 183b36-184a8 », 105-116).

Neuf chercheurs du Centre de Recherche Philologique de l'Université de Lille procèdent à un réexamen de la pensée du présumé fondateur de l'école cynique à partir des témoignages d'Aristote et de Diogène Laërce, “qui sont les seuls à exposer la pensée d'un Antisthène explicitement nommé, d'une manière cohérente et structurée " (118). ("Antisthène: sophistique et cynisme", 117-147). Le témoignage d'Aristote (Mét. $\Delta 29$ et Z 3) concerne exclusivement la conception antisthénienne du langage, alors que la "Vie d'Antisthène " de Diogène Laërce met surtout l'accent sur l'éthique. La grande originalité de cette longue étude, à laquelle un résumé de quelques lignes ne saurait rendre justice, consiste à montrer que la pratique farouche de l'autarcie et de l'individualisme est la conséquence obligée d'une pensée pour laquelle le langage débouche inévitablement sur l'incommunicabilité.

Donnant leur congé aux lieux communs qui font de tous les sophistes des sceptiques, et vice versa, F. Caujolle-Zaslawsky examine à nouveaux frais la question du parallèle entre la sophistique et le scepticisme; mais comme un examen exhaustif déborderait largement le cadre d'un article, l'auteur limite son propos à l'étude du témoignage de Sextus Empiricus sur Protagoras ("Sophistique et scepticisme: l'image de Protagoras dans l'oeuvre de Sextus Empiricus ", 149-165). L'examen de l'interprétation que donne Sextus de la fameuse sentence "l'homme est la mesure de toutes choses" foumit à l'auteur l'occasion de dégager, avec beaucoup de finesse, les principaux points de convergence entre Protagoras et le scepticisme. Sextus se sépare toutefois de Protagoras et des sophistes sur de nombreux points, entre autres en ce qui touche la dialectique, à laquelle il ne reconnaît aucune utilité. 
A. Capizzi propose une stimulante interprétation sociologique de la grande vogue que connurent les sophistes à la fin du Ve siècle. ("La confluence des sophistes à Athènes après la mort de Périclès et ses connexions avec les transformations de la société attique ", 167-177). La mort de Périclès, en 429, mit fin à la mainmise des familles aristocratiques sur les institutions démocratiques. Les nouveaux riches, grands et petits, se coalisèrent alors pour faire main basse sur les partis politiques; mais il manquait à ces parvenus la maîtrise de la parole, maîtrise qui n'avait pas peu contribué à assurer la suprématie de l'aristocratie. Ces nouveaux riches avaient les moyens de s'offrir les cours dispensés par les sophistes, dont l'arrivée à Athènes coïncide précisément avec la mort de Périclès.

L'attitude des sophistes envers la démocratie ne fut certes pas monolithique. Certains d'entre eux, dont Calliclès et Critias, apparaissent en effet comme les fossoyeurs du régime démocratique, alors que d'autres, tel Protagoras, en furent au contraire les chantres. Dans "Sophistique et démocratie " (179-193), R. Müller, membre de l'Académie des Sciences de la R.D.A., ne s'intéresse qu'aux doctrines sophistiques "qui trahissent une attitude positive envers la démocratie et qui, même, ont contribué à motiver et à justifier la démocratie théoriquement et idéologiquement" (180; nos italiques). Les sophistes, idéologues? On ne peut suivre Müller sur ce terrain que si l'on accepte d'emblée cette théorie hautement discutable - et qu'en bon marxiste Müller nous assène comme une vérité établie - selon laquelle la pensée politique des sophistes "est le reflet intellectuel des conflits sociaux ou politiques des Ve et IVe siècles" (180; nos italiques). Nous ne sommes pas très friand de ce type d'étude où les " contradictions sociales " et les "intérêts de classe " (189) sont présentés comme les ressorts et les motivations ultimes de la pensée.

Avec "La certitude subjective inébranlable " (195-208), L. Rosetti tente de montrer qu'un relativisme épistémologique, tel que celui professé par Protagoras, présente l'avantage, considérable, de respecter a priori toute certitude subjective fondée sur les expériences propres à chacun, sans pour autant déboucher sur une impasse, ou encore un pessimisme épistémologique. Cette étude est davantage une réflexion à partir des positions attribuées à Protagoras, qu'une analyse historique portant sur les fragments du célèbre sophiste d'Abdère.

Dans un article dont la lecture laisse pour le moins perplexe, G. Caserno soutient que la conception gorgienne de "logos " vise à conduire l'homme vers "la conscience pleine de ce qu'il est" (213), car "c'est cette pleine conscience seule qui constitue l'être authentique de l'homme " (214; nos italiques). En un raccourci révélateur, Caserno affirme que l'ambition de Gorgias est en somme de "sortir l'homme du train-train quotidien pour rejoindre la conscience authentique » 
(214). Qui ne voit que ces affirmations reviennent à faire de Gorgias un précurseur de l'existentialisme? C'est un anachronisme auquel nous ne pouvons évidemment pas souscrire ("L'amour entre logos et pathos. Quelques considérations sur l'Hélène de Gorgias ", 211220).

Les fragments de Prodicos sont des plus disparates: certains se rapportent à la synonymique, d'autres à l'étude de la nature, et d'autres encore à l'enseignement de la vertu. Cette apparente diversité rend malaisé, sinon impossible, le projet de reconstruire une vision d'ensemble de l'œuvre de Prodicos. Selon J.-P. Dumont (« Prodicos: de la méthode au système ", 221-232), une telle reconstitution exige au préalable que l'on ne confonde pas ce qui relève du système, soit les thèmes abordés (nature, éthique), et ce qui ressortit à la méthode employée (synonymique). L'on retiendra de cette étude que Prodicos ne fut pas un théoricien du langage, mais un praticien.

Pour G. W. Most, la grande nouveauté de l'enseignement sophistique est que "certains sophistes ont assigné une position centrale à l'interprétation des textes littéraires " (237; "Sophistique et herméneutique ", 233-245). Mais si les avancées sophistiques, dans le domaine herméneutique, sont restés sans fruits, c'est qu'elles " ont été systématiquement bloquées par Platon, qui leur opposait une philosophie essentiellement anti-herméneutique " (241). Most s'efforce en effet de prouver que Platon nie aux textes littéraires la capacité d'enseigner la vérité sur quelque sujet que ce soit. Aussi séduisants soient-ils, les arguments de Most ne l'autorisent cependant pas à conclure que la position de Platon «minera les fondements de toute herméneutique pendant plusieurs siècles " (244). Il n'y a rien dans la démonstration de Most qui puisse étayer un jugement aussi préremptoire.

Les trois dernières contributions de ce recueil ne concernent pas directement la première sophistique et ont, pour cette raison, été groupés dans une section intitulée: "Postérité de la sophistique ". Dans la première de ces contributions, $C$. Lanier propose une analyse, dont nous avons grandement apprécié la finesse et la clarté, de l'Éloge de Rome composé par Ælius Aristide, l'un des plus illustres représentants de la seconde sophistique ( Le problème de la constitution mixte chez Elius Aristide ", 247-265). On voit difficilement en quoi l'étude de $C$. MacDonald, consacrée au rôle de la pensée analogique et associative chez Diderot, constitue une illustration d'un avatar de la sophistique ("L'heuristique de la philosophie. Les inventions de Diderot ", 267-290). Les quelques rapprochements maladroits que MacDonald effectue avec la sophistique sont, sinon complètement artificiels, du moins tout à fait accessoires et inessentiels. Dans sa seconde contribution à ce recueil, $M$. Nancy tente de cerner le statut que L. Strauss a réservé à la sophistique ( "La sophistique de Léo Strauss ", 291-300). Ce grand contempteur de la Modernité critique vivement tous ceux qui ont rapproché les 
doctrines sophistiques de l'historicisme et du libéralisme modernes. Et pourtant, la réfutation straussienne de l'historicisme n'est pas sans rappeler les objections qu'Aristote, déjà, élevait contre le relativisme sophistique. Se refusant à comprendre la différence entre les sophistes et les philosophes autrement que les Anciens ne l'ont eux-mêmes comprise, Strauss, à la suite d'Aristote, affirme que la caractéristique essentielle des sophistes est leur proairésis tou biou, leur « choix d'un mode de vie ". Ce choix consiste à accorder plus de prix au prestige qui accompagne la sagesse qu'à la sagesse elle-même.

Enfin une étude bibliographique de la seconde sophistique a été préparée par G. Rocca-Serra (301-314). Bien que ce dernier n'ait pas la prétention d'être exhaustif, nous nous expliquons mal l'absence, sous la rubrique "Galien ", du livre de R.-B. Edlow, Galien on Language and Ambiguity, Leiden: Brill, 1977). Ce livre est en effet du plus haut intérêt pour quiconque s'intéresse à l'analyse des sophismes et à la façon dont l'Antiquité tardive à compris les Réfutations sophistiques d'Aristote.

Le chercheur se félicitera des deux index (auteurs anciens, auteurs et ouvrages modernes) qui complètent ce volume. Plus fondamentalement, nous nous réjouissons de ce que des publications comme Positions de la sophistique et Le plaisir de parler, sans oublier les autres travaux des Cassin, Canto et Nancy, viennent définitivement sonner le glas de l'indifférence que, pendant de trop nombreuses années, la recherche française a témoignée à l'endroit des sophistes et de la sophistique.

Département de philosophie Université de Montréal 